\title{
ANÁLISIS ESTADÍSTICO DEL ÍNDICE DE DIFICULTAD DE LOS ÍTEMS POLITÓMICOS EN LAS
} PRUEBAS PEDAGÓGICAS

\section{STATISTICAL ANALYSIS OF DIFFICULTY INDEX OF POLYCHOTOMOUS ITEMS IN PEDAGOGIC ASSESSMENT}

\author{
Aníbal Cárdenas Ayala \\ Instituto de Investigación de la Facultad de Educación
}

\section{RESUMEN}

En el presente trabajo de investigación, se realizó el análisis estadístico edumétrico de los índices de dificultad de los ítems politómicos en las pruebas pedagógicas que se aplican a los alumnos de las instituciones educativas de la Región Junín. Tuvo como objetivos específicos, establecer los factores ponderativos equivalentes a 0 ó 1, de los calificativos asignados a los ítems politómicos, elaborar algoritmos de cálculo del índice de dificultad de los ítems politómicos y determinar el índice de dificultad de los ítems politómicos en las pruebas pedagógicas que se aplican a los alumnos de las instituciones educativas de la Región Junín. Verificando la hipótesis: existen índices de dificultad de los ítems politómicos en las pruebas pedagógicas que se aplican a los alumnos de las instituciones educativas de la Región Junín. La investigación es del tipo tecnológico-aplicada con un diseño descriptivo simple. Se llevó a cabo en la Universidad Nacional del Centro del Perú, Facultad de Educación, a lo largo de un año calendario comprendido entre el 2011-07-01 y el 2012-06-30. Arribó a la conclusión que, de acuerdo a los análisis y discusión de los resultados, son pertinentes, el cuadro de equivalencias y el algoritmo de cálculo propuesto, para determinar el índice de dificultad de los ítems politómicos de las pruebas pedagógicas que se aplican a los alumnos de las instituciones educativas de la Región Junín.

Palabras clave: Psicometría, Edumetría, Estadística aplicada a la investigación educativa

\begin{abstract}
The investigation made statistical edumetric analysis of difficulty index of polychotomous items in pedagogic assessment are applied to students of the educational institutions of Junín Region. It had as specific objectives, to establish equivalent factors at 0 or 1 of notes assigned to polychotomous items, to elaborate calculation algorithms of difficulty index of polychotomous items, and to determine difficulty index of polychotomous items in pedagogic assessment that are applied to students of the educational institutions of Junín Region. Verifying the hypothesis: difficulty index of polychotomous items exist in pedagogic assessment that are applied to students of the educational institutions of the Junín Region. The investigation is of technological-applied kind with a descriptive simple design. It was made in the Universidad Nacional del Centro del Peru, Education Faculty, throughout a calendar year between 201107-01 and the 2012-06 -30. It arrived to the conclusion that, according to the analyses and discussion of the results, they are pertinent, the square of equivalences and the calculation algorithm proposed, to determine difficulty index of polychotomous in pedagogic assessment that are applied to students of the educational institutions of Junín Region.
\end{abstract}

Key words: Psychometric, Edumetric, Statistic applied to the educational investigation 


\section{INTRODUCCIÓN}

Entre los años 2004 y 2011, el autor del presente proyecto realizó investigaciones centradas en el cálculo de los estadígrafos psicométricos y edumétricos, tales como la validez, confiabilidad, dificultad, sensibilidad e índice de discriminación de los diferentes tipos de instrumentos de recolección de datos, habiendo quedado como sugerencias reiterativas, realizar el análisis estadístico y la interpretación del índice de dificultad de los ítems politómicos en las pruebas pedagógicas y fichas de observación.

Por otro lado, el desarrollo de la asignatura de Estadística Aplicada a la Investigación Educativa, en la Unidad de Post Grado de la Facultad de Educación, implica, entre otros temas, el cálculo del estadígrafo edumétrico: índice de dificultad, para ítems dicotómicos; quedando sin desarrollar, por falta de una sistematización matemática, el algoritmo y la interpretación; para los ítems politómicos de las pruebas pedagógicas y fichas de observación.

Entonces, para poder subsanar, en parte, el inconveniente citado líneas arriba, en el presente trabajo de investigación se diseñaron algoritmos de cálculo del índice de dificultad de los ítems politómicos, de las pruebas pedagógicas y fichas de observación que se aplican a los alumnos de las instituciones educativas de la Región Junín.

\section{METODOLOGÍA DE LA INVESTIGACIÓN}

\section{POBLACIÓN Y MUESTRA DE LA INVESTIGACIÓN}

\section{POBLACIÓN}

Se tomó como población de la investigación a todas las pruebas pedagógicas que aplicaron a sus alumnos, en las diferentes instituciones educativas del nivel secundario de la Región Junín, los estudiantes de la Maestría en Educación, hasta el año académico 2011; los egresados del Pregrado de la Facultad de Educación hasta el año 2011 en sus respectivas tesis; así como a las fichas de observación utilizadas, para evaluaciones procedimentales y actitudinales, por los egresados de la Maestría en Educación, hasta el Año Académico de 2011; las que están consignadas en los anexos de las tesis de grado.

\section{MUESTRA}

Para la elección de la muestra se utilizó un diseño muestral no aleatorio dirigido, de tal manera que, la muestra estuvo conformada por una prueba de pensamiento creativo, una prueba pedagógica, ambas politómicas, que se aplicaron a los alumnos de dos instituciones educativas de la UGELHuancayo y fichas de observación politómica para la evaluación procedimental en la Universidad Peruana "Los Andes".

Nota: Las pruebas pedagógicas y fichas de observación politómicas fueron bastante, escasas.

\section{MÉTODO}

\section{TIPO DE INVESTIGACIÓN}

Dentro del campo de la investigación educativa y de acuerdo a las características de la hipótesis y los objetivos se enmarcará dentro del tipo de investigación tecnológico-aplicada.

\section{MÉTODO BÁSICO DE LA INVESTIGACIÓN}

El método básico fue el descriptivo.

\section{DISEÑO DE LA INVESTIGACIÓN}

El diseño a emplearse en esta investigación será el descriptivo, porque resultará imposible el control experimental riguroso de las variables. Además, este diseño se adecua perfectamente a la presente investigación.

Dentro de los diseños descriptivos se encuentra el diseño descriptivo simple, el que se aplicará para determinar los índices de dificultad de los ítems politómicos en las pruebas pedagógicas que se aplican a los alumnos de las instituciones educativas de la Región Junín.

En el caso concreto de la presente investigación, se aplicarán las pruebas pedagógicas con ítems politómicos, luego con los datos recolectados para 
cada ítem, se calcularán los índices de dificultad, los que serán analizados e interpretados. Sánchez (2006:103).

El diseño se diagrama de la siguiente manera:

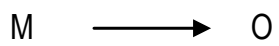

Donde:

M: Pruebas pedagógicas con ítems politómicos que se aplican a los alumnos de las instituciones educativas de la Región Junín.

O: Índice de dificultad de los ítems politómicos de las pruebas que se aplican a los alumnos de las instituciones educativas de Región Junín.

\section{VARIABLES DE LA INVESTIGACIÓN}

\section{VARIABLE INDEPENDIENTE: (VI)}

Ítems politómicos de las pruebas pedagógicas que se aplican a los alumnos de las instituciones educativas de Región Junín.

\section{VARIABLE DEPENDIENTE: (VD)}

Índices de dificultad de los ítems politómicos de las pruebas pedagógicas que se aplican a los alumnos de las instituciones educativas de Región Junín.

\section{INSTRUMENTOS DE RECOPILACIÓN DE DATOS}

Las dos pruebas pedagógicas que se seleccionaron como muestra fueron las que aplicaron a sus alumnos las Profesoras:

- Pianto Cencia, Julia y Tinoco Tovar, Liz (egresadas del pregrado); en la I.E. "La Victoria" - El Tambo; en el Área de C.T.A. $4^{\circ}$ grado de secundaria. Durante el III bimestre del Año Académico Escolar 2006. ANEXO: PRUEBA DE PENSAMIENTO CREATIVO

- Gamero Tello, Victoria, (egresada de maestría); en la I.E. Mariscal Castilla - El Tambo; en la asignatura de Matemática, Nivel Secundario, Tercer Grado. Durante el II bimestre del Año Académico Escolar 2010. ANEXO: PRUEBA PEDAGÓGICA

- Tito Ortega, Erwin (egresado de maestría); en la Universidad Peruana "Los Andes"-Facultad de Ciencias de la Salud, E.A.P. de Medicina Humana - Huancayo; en la asignatura de Ginecología y Obstetricia. Durante el $X$ semestre del Año Académico 2002. ANEXO: FICHAS DE OBSERVACIÓN-EVALUACIÓN PROCEDIMENTAL

Se consideraron a estos instrumentos de recolección de datos, debido a que los resultados de los estadígrafos psicométricos calculados coeficiente de confiabilidad, coeficiente de validezfueron óptimos para los objetivos del presente estudio.

\section{PROCEDIMIENTOS DE RECOPILACIÓN DE DATOS}

Los docentes -mencionados líneas arriba- fueron capacitados (oportunamente) en el diseño, elaboración y aplicación de pruebas. Así como, en el análisis estadístico y el cálculo de los estadígrafos psicométricos de confiabilidad y validez. Los tres instrumentos de recolección de datos, fueron aplicados por los docentes a sus estudiantes, en sus respectivas instituciones educativas; las docentes, Pianto y Tinoco, hicieron la aplicación como parte de su trabajo de tesis de pregrado; la docente Gamero, hizo la aplicación como parte de su labor pedagógica, es más, como parte de un proceso de entrenamiento propio del desarrollo de la asignatura de Estadística Aplicada a la Investigación Educativa, de la Maestría en Educación, para la elaboración de su trabajo de tesis; finalmente, el docente Tito, hizo lo propio como parte de su trabajo de tesis de maestría en Didáctica Universitaria.

\section{TÉCNICAS Y PROCEDIMIENTOS DE PROCESAMIENTO Y ANÁLISIS DE DATOS}

Siendo la investigación de carácter cuantitativo, en primer lugar fueron calculados, mediante procedimientos estadísticos, el paquete estadístico IBM SPSS Statistics 20 y la aplicación de las fórmulas respectivas, los estadígrafos psicométricos de confiabilidad y validez de los tres instrumentos, porque la condición sine qua non de todo instrumento de recolección de datos, previo 
al cálculo de otros estadígrafos psicométricos y edumétricos es que sean confiables y válidos. Los resultados, pueden observarse en los Anexos respectivos.

No obstante, a manera de comentario general se tiene que:

- Todos los coeficientes de confiabilidad de las pruebas se calcularon aplicando la fórmula alfa de crombach (porque ésta se aplica a los ítems politómicos), siendo los resultados mayores 0 iguales que 0,60; por lo que las tres pruebas son confiables.

- Todos los coeficientes de validez de criteriopredictiva, de cada uno de los ítems de las pruebas, fueron calculados aplicando la fórmula de correlación ítem-total, siendo los resultados mayores o iguales que 0,2000; por lo que las tres pruebas son válidas.

\section{ANÁLISIS Y DISCUSIÓN DE RESULTADOS ANÁLISIS PSICOMÉTRICO DE LAS PRUEBAS PEDAGÓGICAS Y FICHA DE OBSERVACIÓN}

Siendo la investigación de carácter cuantitativo, en primer lugar fueron calculados, mediante procedimientos estadísticos, el paquete estadístico IBM SPSS Statistics 20.0 y la aplicación de las fórmulas respectivas, los estadígrafos psicométricos de confiabilidad y validez de los tres instrumentos tomados como espacio muestral de la presente investigación, porque la condición sine qua non de todo instrumento de recolección de datos, es que sea confiable y válido. Los resultados, pueden observarse en los anexos respectivos. (VER ANEXOS)

\section{a). Confiafilidad}

Todos los coeficientes de confiabilidad de las pruebas se calcularon aplicando la fórmula alfa de crombach, siendo todos los resultados, mayores 0 iguales que 0,60; por lo que las tres pruebas son confiables.

$A C=[k / k-1][1-(s i 2 / s t 2)]$

donde:
AC : coeficiente de confiabilidad

k : número de ítems del instrumento.

si2 : sumatoria de varianzas individuales de cada ítem.

st2 : varianza total del instrumento.

\section{b). Validez}

Todos los coeficientes de validez de criterio (predictiva), de cada uno de los ítems de cada una de las pruebas, fueron calculados aplicando la fórmula de correlación item-total, siendo todos los resultados, mayores o iguales que 0,2000; por lo que las tres pruebas son válidas.

$r$ (corregido $)=(r$ (crudo) $. s t-s i) /(s t 2+s i 2-2 r$ (crudo).st si) $1 / 2$

donde:

$r$ (corregido) : coeficiente de validez del ítem. $r$ (crudo) : coeficiente de correlación de pearson.

st : desvío del puntaje total.

si : desvío del ítem.

st2 : varianza del puntaje total.

si2 : varianza del ítem.

\section{ANÁLISIS EDUMÉTRICO DE LAS PRUEBAS PEDAGÓGICAS Y FICHA DE OBSERVACIÓN}

\section{a). PROPUESTA DE ALGORITMOS PARA EL CÁLCULO DEL ÍNDICE DE}

\section{DIFICULTAD DE LOS ÍTEMS POLITÓMICOS DE LOS INSTRUMENTOS DE RECOLECCIÓN DE DATOS}

Conceptualmente un ítem dicotómico es aquel cuya calificación es de cero (0) si está mal respondido, y de uno (1) si está bien respondido; entonces, un ítem politómico será aquel cuya calificación es de cero (0) si está mal respondido, pero si está bien respondido puede tener calificativos de $1 ; 2 ; 3 ; 4$; ... u otros valores enteros 0 inclusive fraccionarios.

El cálculo del índice de dificultad de los ítems mediante la aplicación de la fórmula, implica que todos los ítems de las pruebas sean dicotómicos (0 ó 1) y como el objetivo general del presente trabajo de investigación es identificar los índices de 
dificultad de los ítems politómicos en las pruebas, se propone en primer lugar establecer equivalencias entre ítems politómicos y dicotómicos, así como, factores ponderativos equivalentes a 0 ó 1 , de los calificativos asignados a los ítems politómicos de las pruebas, lo que se plasma en el cuadro de equivalencias, para poder realizar los cálculos del índice de dificultad de cada uno de los ítems politómicos que conforman cada una de las pruebas tomadas como espacio muestral de la presente investigación.

\section{EQUIVALENCIAS DE ÍTEMS POLITÓMICOS CON DICOTÓMICOS}

\section{CUADRO N ${ }^{0} 1$}

\begin{tabular}{|c|c|c|}
\hline İTEM & CALIFICATIVO REAL & $\begin{array}{l}\text { CALIFICATIVO } \\
\text { EQUIVALENTE A } \\
\text { DICOTÓMICO: } \\
\text { factor ponderativo }\end{array}$ \\
\hline DICOTÓMICO & $\begin{array}{l}\text { Mal respondido: } 0 \\
\text { Bien respondido: } 1\end{array}$ & $\begin{array}{l}\text { Mal respondido: } 0 \\
\text { Bien respondido: } 1\end{array}$ \\
\hline POLITÓMICO & $\begin{array}{r}\text { Mal respondido: } 0 \\
\text { Bien respondido: } 1 \\
2\end{array}$ & $\begin{array}{r}\text { Mal respondido: } 0,0 \\
\text { Bien respondido: } 0,5 \\
1,0\end{array}$ \\
\hline POLITÓMICO & $\begin{array}{r}\text { Mal respondido: } 0 \\
\text { Bien respondido: } 1 \\
2\end{array}$ & 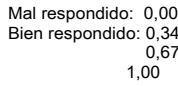 \\
\hline POLITÓMICO & $\begin{array}{r}\text { Mal respondido: } 0 \\
\text { Bien respondido: } 1 \\
2 \\
3\end{array}$ & $\begin{array}{rr}\text { Mal respondido: } & 0,00 \\
\text { Bien respondido: } & 0,25 \\
0,50 \\
0,75 \\
1,00\end{array}$ \\
\hline
\end{tabular}

Fuente: Elaboración del autor (2010)

\section{b). CÁLCULO DE LOS ÍNDICES DE DIFICULTAD}

\section{Índice de dificultad de cada uno de los ítems}

El cálculo, se realiza a través de la fórmula:

\section{Índice de dificultad (ID) $=\Sigma \mathrm{RC} / \mathrm{n}$}

Donde:

प RC : sumatoria de respuestas correctas $\left(\mathrm{N}^{\circ}\right.$ de participantes que respondieron bien el ítem analizado).

$\mathrm{n}: \mathrm{N}^{0}$ total de participantes evaluados.

Esta fórmula se aplica directamente cuando los ítems son dicotómicos, es decir, los ítems han sido totalmente respondidos; pero cuando los ítems son politómicos, primero se tienen que establecer las equivalencias de ítem politómico a dicotómico (con el cuadro de equivalencias propuesto), porque un ítem puede haber sido parcialmente respondido; lo que implica que a la matriz de ítems politómicos reales (con valores de $0 ; 1 ; 2 ; 3$ ó 4) se la modifique para obtener una nueva matriz de ítems equivalentes a dicotómicos, mediante una multiplicación del calificativo real del ítem por el factor ponderativo, lo que da como resultado ítems ponderados, así como un calificativo total modificado. En esta nueva matriz de ítems ponderados, los valores, ahora, están comprendidos entre cero (0) y uno (1), tal como se aprecian en el cuadro de equivalencias. (VER ANEXOS)

Con estas modificaciones, los componentes de la fórmula para el cálculo del índice de dificultad, quedan como:

\section{Índice de dificultad (ID) $=\Sigma \mathrm{RCP} / \mathrm{n}$}

Donde:

$\Sigma$ RCP: sumatoria de los ítems total o parcialmente respondidos (valores

ponderados en función al $\mathrm{N}^{0}$ de participantes que respondieron bien, de

manera total o parcial el ítem analizado).

$\mathrm{n}: \mathrm{N}^{0}$ total de participantes evaluados.

El valor obtenido debe ser positivo y estar comprendido entre 0,00 y 1,00 para discernir el grado de dificultad del ítem.

\section{Índice de dificultad de todo el instrumento}

El cálculo para toda la prueba, se realiza a través de la fórmula:

Índice de dificultad (IDT) = X/ Pmáx (fórmula No1)

Donde:

$X$ : Promedio de los puntajes totales obtenidos por los participantes en la prueba

Pmáx: Puntaje máximo que vale la prueba. 
Esta fórmula se aplica directamente cuando los ítems son dicotómicos, es decir, los ítems han sido totalmente respondidos, pero cuando los ítems son politómicos, al igual que para el cálculo que se hizo para cada ítem, primero se tienen que establecer las equivalencias de ítem politómico a dicotómico (con el cuadro de equivalencias propuesto), porque un ítem puede haber sido parcialmente respondido; lo que implica que a la matriz de ítems politómicos reales (con valores de $0 ; 1 ; 2 ; 3$ ó 4) se la modifique para obtener una nueva matriz de ítems equivalentes a dicotómicos, mediante la multiplicación del calificativo real del ítem por el factor ponderativo, lo que da como resultado ítems ponderados, así como un calificativo total modificado. En esta nueva matriz de ítems ponderados, los valores, ahora, están comprendidos entre cero (0) y uno (1), tal como se aprecian en el cuadro de equivalencias. (VER ANEXOS)

Con estas modificaciones, los componentes de la fórmula para el cálculo del índice de dificultad para toda la prueba, queda como:

\section{Índice de dificultad (IDT) = X/ Pmáx (fórmula N²-modificada)}

\section{Donde:}

$X$ : Promedio de los puntajes totales modificados por la ponderación, obtenidos por los participantes en la prueba

Pmáx: Puntaje máximo ponderado que vale la prueba.

Nota: Se puede calcular el Índice de Dificultad de la prueba, aplicando directamente la fórmula $\mathrm{N}^{0} 1$, pero es mejor aplicar la fórmula $\mathrm{N}^{0} 2$, porque a veces puede haber incrementos en el índice de dificultad, calculados con la primera. No perder de vista de que se están tomando hasta dos cifras significativas en los decimales.

\section{CLASIFICADOR DE LOS ÍTEMS POR EL ÍNDICE DEDIFICULTAD}

CUADRO No2

\begin{tabular}{lc}
\hline CLASIFICACIÓN & VALOR DEL ÍNDICE DE \\
DEL ÍTEM & DIFICULTAD DEL ÍTEM \\
\hline Muy fácil & De 0,81 a 1,00 \\
Relativamente fácil & De 0,66 a 0,80 \\
Dificultad adecuada & De 0,51 a 0,65 \\
Relativamente difícil & De 0,31 a 0,50 \\
Difícil & De 0,11 a 0,30 \\
Muy difícil & De 0,00 a 0,10 \\
\hline
\end{tabular}

Fuente: adaptado de Cortada 1999

\section{RESULTADOS Y DISCUSIÓN}

Los índices de dificultad de cada uno de los ítems de los instrumentos de recolección de datos seleccionados como parte de la muestra fueron calculados mediante la aplicación de la fórmula y con la ayuda del paquete informático estadístico IBM SPSS Statistics 20. (VER ANEXOS)

\section{a). RESULTADOS Y DISCUSIÓN PARA LA PRUEBA DE CREATIVIDAD}

\section{CUADRO No3}

Como se puede apreciar los índices de dificultad de los ítems, de acuerdo al cuadro clasificador, están comprendidos como relativamente fáciles (8 ítems) y muy fáciles (1 ítem) y tan sólo el ítem 10 es difícil; es más, la prueba en su totalidad tiene una dificultad adecuada.

\section{b). RESULTADOS Y DISCUSIÓN PARA LA PRUEBA PEDAGÓGICA}

\section{CUADRO N4}

\begin{tabular}{lcc}
\hline ITEMS & $\begin{array}{c}\text { ÍNDICE DE } \\
\text { DIFICULTAD }\end{array}$ & COMENTARIO \\
\hline ITEM 01 & 0,83 & Muy fácil \\
ITEM 02 & 0,81 & Muy fácil \\
ITEM 03 & 0,38 & Relativamente dificil \\
ITEM 04 & 0,38 & Relativamente dificil \\
ITEM 05 & 0,52 & Dificultad adecuada \\
ITEM 06 & 0,59 & Dificultad adecuada \\
ITEM 07 & 0,76 & Relativamente fácil \\
ITEM 08 & 0,59 & Dificultad adecuada \\
ITEM 09 & 0,34 & Relativamente difícil \\
ITEM 10 & 0,34 & Relativamente difíil \\
TOTAL PRUEBA & 0,54 & DIFICULTAD ADECUADA \\
\hline
\end{tabular}

Como se puede apreciar los índices de dificultad de los ítems, de acuerdo al cuadro clasificador, están comprendidos como muy fáciles (2 ítems), relativamente difíciles (4 ítems), relativamente 
fáciles (1 ítem) y de dificultad adecuada (3 ítems); es más, la prueba en su totalidad tiene una dificultad adecuada.

c). RESULTADOS Y DISCUSIÓN PARA LA FICHAS DE OBSERVACIÓN-EVALUACIÓN PROCEDIMENTAL

\section{CUADRO N ${ }^{\circ} 5$}

\begin{tabular}{ccc}
\hline ITEMS & $\begin{array}{c}\text { ÍNDICE DE } \\
\text { DIFICULTAD }\end{array}$ & COMENTARIO \\
\hline ITEM 01 & 0,80 & Relativamente fácil \\
ITEM 02 & 0.83 & Muy fácil \\
ITEM 03 & 0,83 & Muy fácil \\
ITEM 04 & 0,78 & Relativamente fácil \\
ITEM 05 & 0,85 & Muy fácil \\
ITEM 06 & 0,88 & Muy fácil \\
ITEM 07 & 0,85 & Muy fácil \\
ITEM 08 & 0,85 & Muy fácil \\
ITEM 09 & 0,80 & Relativamente fácil \\
ITEM 10 & 0,70 & Relativamente fácil \\
ITEM 11 & 0,75 & Relativamente fácil \\
ITEM 12 & 0,85 & Muy fácil \\
ITEM 13 & 0,73 & Relativamente fácil \\
ITEM 14 & 0,75 & Relativamente fácil \\
ITEM 15 & 0,80 & Relativamente fácil \\
ITEM 16 & 0,83 & Muy fácil \\
ITEM 17 & 0,80 & Relativamente fácil \\
ITEM 18 & 0,80 & Relativamente fácil \\
ITEM 19 & 0,85 & Muy fácil \\
ITEM 20 & 0,80 & Relativamente fácil \\
TOTAL PRUEBA & 0,81 & MUY FÁCIL \\
\hline
\end{tabular}

Como se puede apreciar los índices de dificultad de los ítems, de acuerdo al cuadro clasificador, están comprendidos como muy fáciles (9 ítems), relativamente fáciles (11 ítems); es más, la ficha de observación en su totalidad es muy fácil.

\section{d). DISCUSIÓN FINAL DE RESULTADOS}

Todos los instrumentos de recolección de datos, específicamente las pruebas pedagógicas con ítems dicotómicos, deben de tener un índice de dificultad adecuado, es decir, tener un índice de dificultad comprendido entre 0,51 y 0,65 Nunnally / Berstein (1995).

La facilidad para un ítem dicotómico se calcula contabilizando las respuestas acertadas de los participantes, en la prueba, que lo resolvieron bien; mientras más participantes lo resolvieron bien, más fácil es el ítem y mientras menos lo resolvieran más difícil, Shiefelbeim (1995).

La forma de cálculo del índice de dificultad para un ítem dicotómico, también es posible hacerla para un ítem politómico, sólo que hay que tener en cuenta el grado en que fueron resueltos, éste es gradual a la intensidad de cómo fueron resueltos, siendo así, si la resolución fue total entonces el ítem es fácil, pero si la resolución fue parcial la dificultad del ítem varía, haciéndose desde menos fácil a más difícil, hasta llegar al punto de no ser resuelto, en otras palabras difícil; este efecto se pudo medir con el cuadro de equivalencias de ítems politómicos con dicotómicos, previo al empleo de fórmula pertinente; concluyendo que es posible calcular el índice de dificultad de los ítems politómicos.

\section{CONCLUSIONES}

- Se elaboró un cuadro de equivalencias de ítems politómicos con ítems dicotómicos, mediante la aplicación de factores ponderativos, de las pruebas pedagógicas que se aplican a los alumnos de las instituciones educativas de la Región Junín.

- Se diseñaron algoritmos para el cálculo de los índices de dificultad de los ítems Politómicos, de las pruebas pedagógicas que se aplican a los alumnos de las instituciones educativas de la Región Junín.

- Se determinaron los índices de dificultad de los ítems politómicos de las pruebas pedagógicas que se aplican a los alumnos de las instituciones educativas de la Región Junín.

- De acuerdo a los análisis y discusión de los resultados, son pertinentes, el cuadro de equivalencias y el algoritmo de cálculo propuesto, para determinar el índice de dificultad de los ítems politómicos de las pruebas pedagógicas que se aplican a los alumnos de las instituciones educativas de la Región Junín.

\begin{tabular}{lcc}
\hline ITEMS & $\begin{array}{c}\text { INDICE DE } \\
\text { DIFICULTAD }\end{array}$ & COMENTARIO \\
\hline ITEM 01 & 0,74 & Relativamente fácil \\
ITEM 02 0,68 & Relativamente fácil \\
ITEM 03 & 0,66 & Relativamente fácil \\
ITEM 04 04 & Relativamente fácil \\
ITEM 05 & 0,74 & Relativamente fácil \\
ITEM 06 & 0,76 & Relativamente fácil \\
ITEM 07 & 0,72 & Relativamente fácil \\
ITEM 08 & 0,68 & Muy fácil \\
ITEM 09 & 0,82 & Relativamente fácil \\
ITEM 10 & 0,66 & Difícil \\
TOTAL PRUEBA & 0,12 & DIFICULTAD ADECUADA
\end{tabular}




\section{REFERENCIAS BIBLIOGRÁFICAS}

1. Aiken, L. Test psicológicos y evaluación. México D.F., México: Editorial Prentice Hall Hispanoamericana, 1995.

2. Ary; Jacobs y Razavieh. Introducción a la investigación pedagógica. México D.F.,México: Editorial McGraw-Hill, 1992.

3. Cárdenas Ayala, A. Pertinencia de los Instrumentos de recolección de datos con los métodos estadísticos de confiabilidad. (I.I. FPH-UNCP, trabajo de investigación inédito). Huancayo, Perú, 2007.

4. Cárdenas Ayala, A. Análisis estadístico de los instrumentos de recolección de datos a través de los estadígrafos de deformación y apuntamiento. (I.I.FPH-UNCP, trabajo de investigación inédito). Huancayo, Perú, 2008.

5. Cárdenas Ayala, A. Poder discriminativo, nivel de exigencia y capacidad predictiva de los exámenes de selección, I.I.FPH-UNCP, trabajo de investigación inédito, Huancayo, Perú, 2009.

6. Cárdenas Ayala, A. Relación entre la validez, el poder discriminativo y nivel de dificultad de las pruebas pedagógicas. Horizonte de la ciencia, 01, 109-119. Huancayo, Perú, 2010.

7. Cárdenas Ayala, A. Índice de discriminación de los ítems politómicos en las pruebas pedagógicas. Prospectiva universitaria, V- 8, 01, 119-126. Huancayo, Perú, 2011.

8. Davis, F. Analyse des items. Lovaina, París: Nauwelaerts, 1989.

9. Delgado; Escurra y Torres. La medición en psicología y educación. Lima,Perú: Editorial Hozlo S.R.L., 2006.

10. Fermín, M. La evaluación, los exámenes y las calificaciones. Buenos Aires, Argentina: Editorial Kapeluz, 1995.

11. Gonzalvo, G. Diccionario de metodología estadística. Madrid, España: Ediciones Morata S.A., 1988.
12. Hambleton y Novik. Toward an integration of theory and metod for criterion referenced test. New York, USA: Editoral Measurmet, 1989.

13. Hernández; Fernández y Baptista. Metodología de la investigación. México: Editorial McGraw-Hill, 2003.

14. Nunnally y Bernstein. Teoría psicométrica. México: Editorial Mc Graw-Hill, 1995.

15. Quezada, N. Metodología de la investigaciónEstadística aplicada en la investigación. Lima, Perú: Empresa Editora MACRO, 2010.

16. Sánchez y Reyes. Metodología y Diseños en la Investigación Científica. Lima, Perú: Editorial Visión Universitaria, 2006.

17. Schiefelbein, E. Teoría, técnicas, procesos y casos en el planeamiento de la educación. Buenos Aires, Argentina: Editorial Ateneo, 1999.

18. Santisteban, C. Principios de psicometría. Madrid, España: Editorial Síntesis, 2009.

19. Tavella, N. Análisis de los ítemes en la costrucción de instrumentos, 1991.

20. Psicométricos. México: Editorial Trillas.

21. Tembrick, T. Evaluación. Madrid, España: Editorial Narcea, 1981.

22. Thorndikee y Hagen. Medición y evaluación en psicología y educación. México: Editorial Trillas, 1991.

23. Visauta y Martori i Cañas. Análisis estadístico con SPSS para windows, (Volumen II). Madrid, España: Editorial McGraw-Hill, 2003.

\section{REFERENCIAS DE INTERNET}

Quantitative data processing: Churchman / Ackoff I Arnoff

http://www. yale university.research center.org.usa Septiembre, 2007 